\title{
Temporal phosphate gradients reveal diverse acclimation responses in phytoplankton phosphate uptake
}

\author{
Carlos Cáceres $^{1,2} \cdot$ Sofie Spatharis $\mathbb{C}^{3,4} \cdot$ Eirini Kaiserli ${ }^{5} \cdot$ Evangelia Smeti $^{6} \cdot$ Hugh Flowers $^{7} \cdot$ Juan A. Bonachela $\mathbb{C}^{1,8}$
}

Received: 10 December 2018 / Revised: 11 June 2019 / Accepted: 28 June 2019 / Published online: 26 July 2019

(c) The Author(s), under exclusive licence to International Society for Microbial Ecology 2019

\begin{abstract}
Phytoplankton face environmental nutrient variations that occur in the dynamic upper layers of the ocean. Phytoplankton cells are able to rapidly acclimate to nutrient fluctuations by adjusting their nutrient-uptake system and metabolism. Disentangling these acclimation responses is a critical step in bridging the gap between phytoplankton cellular physiology and community ecology. Here, we analyzed the dynamics of phosphate $(\mathrm{P})$ uptake acclimation responses along different $\mathrm{P}$ temporal gradients by using batch cultures of the diatom Phaeodactylum tricornutum. We employed a multidisciplinary approach that combined nutrient-uptake bioassays, transcriptomic analysis, and mathematical models. Our results indicated that cells increase their maximum nutrient-uptake rate $\left(V_{\max }\right)$ both in response to $\mathrm{P}$ pulses and strong phosphorus limitation. The upregulation of three genes coding for different $\mathrm{P}$ transporters in cells experiencing low intracellular phosphorus levels supported some of the observed $V_{\max }$ variations. In addition, our mathematical model reproduced the empirical $V_{\max }$ patterns by including two types of $\mathrm{P}$ transporters upregulated at medium-high environmental and low intracellular phosphorus levels, respectively. Our results highlight the existence of a sequence of acclimation stages along the phosphate continuum that can be understood as a succession of acclimation responses. We provide a novel conceptual framework that can contribute to integrating and understanding the dynamics and wide diversity of acclimation responses developed by phytoplankton.
\end{abstract}

\section{Introduction}

Phytoplankton play a key role in global biogeochemical cycles, being responsible for $\sim 40 \%$ of the global primary production and fueling oceanic food webs [1-3].

Supplementary information The online version of this article (https:// doi.org/10.1038/s41396-019-0473-1) contains supplementary material, which is available to authorized users.

$\triangle$ Carlos Cáceres

caceres.30@osu.edu

$\triangle$ Juan A. Bonachela

juan.bonachela@rutgers.edu

1 Department of Mathematics and Statistics, University of Strathclyde, Livingstone Tower, 26 Richmond St., Glasgow, Scotland G1 1XH, UK

2 Schiermeier Olentangy River Wetland Research Park, School of Environment and Natural Resources, The Ohio State University, Columbus, OH 43202, USA

3 Institute of Biodiversity, Animal Health and Comparative Medicine, University of Glasgow, Glasgow, Scotland G12 8QQ, UK
Phytoplankton grow in the euphotic layers of aquatic environments, where concentrations of inorganic nutrients fluctuate widely. Such fluctuations affect phytoplankton community composition by favoring species and ecotypes with eco-physiological traits best suited to exploit available nutrients [4]. Nutrient changes also trigger responses at the cellular level, as phytoplankton modify their ecophysiological traits, including those related to nutrient uptake [5-7]. Due to the short time required for these

4 School of Life Sciences, University of Glasgow, Glasgow, Scotland G12 8QQ, UK

5 Institute of Molecular, Cell and Systems Biology, College of Medical, Veterinary and Life Sciences, University of Glasgow, Glasgow G12 8QQ, UK

6 Hellenic Centre for Marine Research, Institute of Marine Biological Resources and Inland Waters, 46.7 km Athens-Sounio Ave., Anavyssos 19013, Greece

7 Department of Chemistry, University of Glasgow, Glasgow, Scotland G12 8QQ, UK

8 Department of Ecology, Evolution, and Natural Resources, Rutgers University, 14 College Farm Road, New Brunswick, NJ 08901, USA 
individual level acclimation changes to occur, these responses can be relevant for exploiting a variety of conditions along the nutrient continuum, from oligotrophic environments to sharp and/or short-lived nutrient pulses [5, 6, 8-10]. In spite of their importance, acclimation dynamics along nutrient spatiotemporal gradients are still poorly understood.

The study of phytoplankton nutrient-uptake acclimation responses associated with changes in nutrient levels has been traditionally based on the variations of the maximum nutrient-uptake rate $\left(V_{\max }\right)$ and the half saturation constant for nutrient uptake $(K)$ measured from nutrient-uptake bioassays [6, 11, 12]. $V_{\max }$ and $K$ are the parameters of the Michaelis-Menten equation, commonly employed to describe nutrient-uptake rate as a function of the external nutrient concentration $[13,14]$, and are linked to key ecophysiological traits $[15,16]: V_{\max }$ determines the potential of the cell to take up nutrients and is related to the number of nutrient-uptake sites present in the cell membrane [5, 17]. $K$ and the ratio between $V_{\max }$ and $K$ [18] indicate the efficiency of the process and define the ability of the cell to exploit oligotrophic environments (for constant $V_{\max }$, the lower the $K$ the higher the nutrient uptake). Existing literature has reported, however, contradictory relationships between $V_{\max }$ and the limiting nutrient concentration.

Most empirical studies show a higher $V_{\max }$ in cultures where nutrients are depleted, enabling the cells to improve nutrient intake abilities [6, 11, 12, 19]. In contrast to these observations, some investigations reported a higher $V_{\max }$ at high nutrient levels, suggesting that cells could take advantage of the often brief high nutrient concentrations $[5,20]$. Regarding variations in $K$, these have been linked to the existence of different types of nutrient transporters, each of which can display different nutrient-uptake rates and regulation mechanisms [20-23]. Transporters have consequently been divided in high and low-affinity groups attending to their half saturation constant [23]. Recent transcriptomic analyses confirmed the existence of different transporters for the same nutrient, with likely different $V_{\text {max }}$ and $K$ values and regulation mechanisms [24-28]. Such transporter diversity might contribute to the aforementioned variation in $V_{\max }$ patterns.

Despite their potential relevance for the understanding of phytoplankton acclimation, there is a lack of empirical work combining $V_{\max }$ and $K$ measurements with transcriptomic analysis for identifying the mechanisms underlying $V_{\max }$ and $K$ variations along the nutrient spatiotemporal gradient. Furthermore, most $V_{\max }$ studies have been addressed using steady state cultures, i.e., chemostats, or batch cultures sampled at unique time shots (but see ref. [17]), which has limited the comprehension of the dynamics of the acclimation process and the development of mechanistic mathematical models. Indeed, no existing theoretical framework integrates the wide diversity of acclimation patterns occurring along the spatiotemporal nutrient gradient. Theories considering acclimation responses typically focus on the negative relationship between $V_{\max }$ and nutrient concentrations, mostly using phenomenological expressions [7, 15, 29].

Here, we aim to fill this gap by (i) understanding mechanistically the dynamics of phytoplankton phosphate uptake acclimation along temporal gradients of phosphate (hereafter P), and (ii) providing a theoretical framework able to integrate the wide diversity of nutrient-uptake acclimation responses and consider the temporal dimension of the acclimation process. To this end, we followed a multidisciplinary approach combining $\mathrm{P}$ uptake bioassays, transcriptomic analysis, and mathematical models [30]. We monitored for the first time the dynamics (i.e., behavior through several consecutive time points) of $V_{\max }$ and $K$ for phosphate along different $\mathrm{P}$ gradients by using batch cultures of the diatom Phaeodactylum tricornutum supplied with different pulses of $\mathrm{P}$. We also measured the expression of genes coding for $\mathrm{P}$ transporters and proteins involved in phosphorus metabolism. Finally, we developed a mechanistic model that integrates the specific temporal patterns of acclimation observed in our experiments in a continuous moving picture [30]. Building on previous work [31], we considered two kinds of nutrient transporters operating in parallel [32]. The combination of our empirical and theoretical results revealed a sequence of acclimation responses along the temporal nutrient gradients that includes both positive and negative $V_{\max }$-nutrient relationships. Our results provide a novel ecological framework that can contribute to understanding and constraining the dynamics and wide diversity of nutrient-uptake acclimation responses displayed by phytoplankton.

\section{Material and methods}

\section{Phytoplankton cultures and experimental design}

Strain 1052/1A (isolated off Plymouth, UK) of the diatom $P$. tricornutum was obtained from the culture collection of the Scottish Association of Marine Sciences (SAMS). $P$. tricornutum is an emerging model species in molecular biology. It mainly grows in coastal waters from tropical and temperate latitudes, including estuaries and areas affected by tidal mixing. Some of these environments show strong and sharp nutrient variations [33-35]. This makes $P$. tricornutum a good candidate for the present study. We inoculated 10 flasks with $5 \times 10^{4}$ cells $\mathrm{mL}^{-1}$ and $\mathrm{F} / 2$ medium $(200 \mathrm{~mL})$ with a reduced concentration of $\mathrm{P}(3 \mu \mathrm{M}$ instead of the 36.2 $\mu \mathrm{M}$ from $\mathrm{F} / 2$ ) to ensure phosphorus limitation. Based on Zhang et al. [8], we also added a mix of antibiotics 
containing penicillin $\left(1.00 \mathrm{gL}^{-1}\right)$, kanamycin $\left(0.50 \mathrm{gL}^{-1}\right)$, and neomycin $\left(0.25 \mathrm{gL}^{-1}\right)$ to prevent the growth of bacteria. We used a light intensity of $20 \mu \mathrm{mol} \mathrm{m} \mathrm{m}^{-2} \mathrm{~s}^{-1}$. Temperature was set at $20^{\circ} \mathrm{C}$ and shaking at $140 \mathrm{rpm}$.

Five days after starting the cultures, we collected different aliquots from one of the ten flasks (see Fig. S1 for a schematic diagram of the experimental design). These aliquots were employed for measuring $V_{\max }, K$, intracellular phosphorus content (i.e., quota, $Q$ ), and expression of genes related to phosphorus metabolism (see below). One day later (day 6), when cells were in a transition to a stationary phase (i.e., phosphorus limited but not totally starved; Fig. S2), we added nutrient pulses (F/2 medium with different levels of $\mathrm{P}$ ) to the nine remaining flasks: three flasks were supplied with no $\mathrm{P}$ (reference treatment, $P_{\text {ref }}$ ), three with $3 \mu \mathrm{M}$ P (low P pulse treatment, $P_{\text {low }}$ ), and three with $15 \mu \mathrm{M}$ P (high $\mathrm{P}$ pulse treatment, $P_{\text {high }}$ ). One flask from each treatment was sampled and measurements performed $3.5,28$, and $100 \mathrm{~h}$ after adding the corresponding pulse of nutrients, so that we obtained a time series for the three different $\mathrm{P}$ addition levels (Fig. S1). The whole experiment was carried out three times with 1 week in between to generate three replicates (Fig. S1). In order to keep the metabolic state of the cells as similar as possible among the three replicates, the cells inoculated to each experiment came from cultures sequentially initiated (also with one week in between). We alternated the time at which each $\mathrm{P}$ pulse was added among experimental replicates to prevent a spurious association between P pulse and uptake traits due to the effect of the cell dial cycle. For similar reasons, we used continuous (i.e., $24 \mathrm{~h}$ ) illumination.

\section{Phosphate uptake bioassays}

We estimated $V_{\max }$ and effective $K\left(K_{\text {eff }}\right)$ by performing Puptake bioassays based on Lomas et al. [10]. We incubated solutions $(10 \mathrm{~mL})$ containing 7500 cells $\mathrm{mL}^{-1}$, $0.20 \mu \mathrm{Ci}$ of $\mathrm{H}_{3}{ }^{33} \mathrm{PO}_{4}$, and different amounts of nonradioactive $\mathrm{H}_{3} \mathrm{PO}_{4}$ (from 0.1 to $7 \mu \mathrm{M}$ ) for $20 \mathrm{~min}$, so that each solution contained a different concentration of nonradioactive $\mathrm{H}_{3} \mathrm{PO}_{4}$ (see SI for further details). Samples were filtered and the radioactivity in the filters was measured using a scintillation counter. The rate of P-uptake rate $\left(V\right.$, fmol cell $\left.{ }^{-1} \mathrm{~d}^{-1}\right)$ was then estimated from the measured radioactivity and the $\mathrm{P}$ concentration during incubations (Eq. [S2]). We fitted the Michaelis-Menten function (Eq. [S1]) to P-uptake observations using $\mathrm{R}$ software to obtain the curve (Fig. S3) and its associated parameters, $V_{\max }$ and $K_{\text {eff }}$. We repeated this process with the different cultures (i.e., flasks) to estimate $V_{\max }$ and $K_{\text {eff }}$ in each $\mathrm{P}$ pulse treatment $24 \mathrm{~h}$ before and $3.5,28$, and $100 \mathrm{~h}$ after $\mathrm{P}$ pulses (Fig. S1).

\section{Intracellular phosphorus measurement}

The culture aliquots $(25 \mathrm{~mL})$ were filtered through a $47 \mathrm{~mm}$ pre-combusted glass fiber filter with $1.2 \mu \mathrm{m}$ of pore size. The filters were then washed with salt water and an oxalate solution to remove any $\mathrm{P}$ adsorbed to the cells [36]. Subsequently, we digested the organic phosphorus in the filters by using concentrated nitric acid (see SI). Filters washed with salt water and oxalate solution were used as a negative control (blank). The phosphorus quota ( $\mathrm{fmol} \mathrm{cell}{ }^{-1}$ ) was estimated from the $\mathrm{P}$ concentration in the digested solution (Eq. [S3]), which was measured using a flow injection autoanalyzer.

\section{Gene expression analysis: RNA extraction and quantitative real-time PCR analysis (qRT-PCR)}

RNA extraction was performed on a $150 \mathrm{~mL}$ cell culture aliquot that was centrifuged at $4000 \times g$ for $15 \mathrm{~min}$. The supernatant was removed and the cells (pellet) were stored in $-80^{\circ} \mathrm{C}$. Total RNA was extracted using the RNAeasy plant mini kit (Qiagen) and cDNA was synthesized from $250 \mathrm{ng}$ of RNA using the QuantiTect reverse transcription kit (Qiagen). Quantitative RT-PCR was performed to analyze the expression of the following genes: HISTONE 4 (H4) (ref. [37]), Na/P co-transporters 47666 ( $\mathrm{Na} / \mathrm{Pi}$ 47666) and 47667 (Na/Pi 47667), P transporter 39515 ( $P i$ 39515), Alkaline phosphatase 49678 (AP 49678), and Carbamoyl phosphate synthetase 1583 (CPS 1583) (ref. [28]; see SI for further details). Normalization of the quantitative real-time PCR data was calculated by geometric averaging of the internal reference gene $H 4$ (refs. [38, 39]).

\section{Statistical analyses}

\section{Uptake traits, quota, and cell abundance}

The effect of P pulses $\left(P_{\text {ref }}, P_{\text {low }}\right.$, and $\left.P_{\text {high }}\right)$ on the temporal dynamics of $V_{\text {max }}, K_{\text {eff }}, V_{\text {max }}: K_{\text {eff }}, Q$, and $N$ was tested with analysis of variance (ANOVA) on a linear mixed effects model including $\mathrm{P}$ pulse and time as fixed factors and experiment as a random factor (each experiment constitutes a separate set of measurements; see SI for a detailed description of the model). Data were fourth-root transformed to approximate to homoscedasticity and normality assumptions (Fig. S4; ref. [40]). The occurrence of differences in the response variables among the three $\mathrm{P}$ temporal gradients corresponding to the three nutrient pulses would be reflected by a significant effect of the factor $P$ pulse or an interaction between the factors $\mathrm{P}$ pulse and Time. Models were fitted by employing the nlme $\mathrm{R}$ package $[41,42]$. To test for statistical differences between specific levels of fixed factors, we conducted pairwise comparisons using the 
emmeans $\mathrm{R}$ package [43] and correcting p-values according to the Benjamini and Hochberg [44] procedure (see SI for further details).

\section{Gene expression}

The relative expression of each gene (\%) was estimated by using $P_{\text {ref }} 3.5 \mathrm{~h}$ after the pulse as a reference. Since we were only able to extract RNA from two experiments, we focused on whether the response presented the same trend across different $\mathrm{P}$ pulses and time points.

\section{Mathematical model for phosphate uptake acclimation}

Our mechanistic eco-physiological model describing acclimation responses uses a modified Droop model [14] to represent the link between the specific growth rate $\left(\mu, \mathrm{d}^{-1}\right)$ and the intracellular content or quota $\left(\mathrm{fmol} \mathrm{cell}{ }^{-1}\right.$ ) of the limiting nutrient, in our case phosphorus:

$\mu(Q, N)=\mu_{\infty}\left(1-\frac{Q_{\min }}{Q}\right)-\alpha N^{2}$,

where $\mu_{\infty}$ is the growth rate for infinite $Q, Q_{\min }$ is the minimum quota required for growth (i.e., $Q$ when $\mu=0$ ), and $N$ is the abundance of cells (cells $\mathrm{L}^{-1}$ ). Differently from the usual Droop equation, we included a $\alpha N^{2}$ term representing generically density-dependent effects such as mutual shading or the release of secondary metabolites to the medium that may occur, ultimately impacting growth at the cellular level $([45,46]$; see SI for further details). The temporal dynamics of the population abundance are given by:

$\frac{d N}{d t}=\mu(Q, N) N$

where we have considered any source of mortality negligible. The change with time of $Q$, in turn, depends on the balance between phosphate uptake rate $(V)$ and cell division (note that cell division leads to a distribution of the cell's phosphorus content):

$\frac{d Q}{d t}=V-\mu(Q, N) Q$

$V\left(\right.$ fmol cell $\left.{ }^{-1} \mathrm{~d}^{-1}\right)$ is represented as a function of the external $\mathrm{P}$ concentration $\left(\mu \mathrm{mol} \mathrm{L} \mathrm{L}^{-1}\right.$ ) by using a modified version of the Michaelis-Menten's equation that considers the potential effect that the simultaneous operation of several types of transporters may have on the uptake of the focal nutrient. Thus, $V$ is estimated by adding the uptake associated with all transporter types for P [32]:

$V=\sum_{i=1}^{j} V_{\max , i} \frac{P}{P+K_{\mathrm{eff}, i}}$

where $j$ is the number of different transporter types, and $V_{\max , i}$ and $K_{\mathrm{eff}, i}$ are the maximum uptake rate and effective half saturation constant linked to transporter type $i$ (see SI).

We considered two types of $\mathrm{P}$ transporters $(j=2)$ : transporter $_{1}$ and transporter 2 , which represent high-affinity (low $K$ ) and low-affinity (high $K$ ) transporters, respectively (see SI and ref. [23]). The effective $V_{\max }$ measured in the uptake bioassays effectively includes the transporterspecific $V_{\max , i}$, in turn linked to the abundance (per cell) of each type of transporter $i\left(n_{i}\right)$ (see SI for further details).

Because the focal acclimation response here is the cell's ability to regulate the number of transporters of each type as nutrient availability changes, we represented explicitly the dynamics of the abundance of each type of transporter as a balance between the synthesis and decrease in number due to cell division. Based on Lomas et al. [10], transporter ${ }_{1}$ is upregulated at low intracellular P levels, which facilitates survival in oligotrophic environments:

$$
\begin{gathered}
\frac{d n_{1}(t)}{d t}=\nu_{1} H\left(c_{H}-\sum_{i=1}^{j} A_{r e l, i}(t)\right) \\
F\left(\frac{c_{F, a} Q_{\min }-Q(t)}{c_{F, a} Q_{\min }-Q_{\min }}\right)-n_{1}(t) \mu(Q, N),
\end{gathered}
$$

where $v_{1}$ is the maximum synthesis rate of transporter ${ }_{1}$ (transporters $\mathrm{d}^{-1}$ ). $F$ is a sigmoid function representing the expression of transporter:

$$
F\left(\frac{c_{F, a} Q_{\min }-Q(t)}{c_{F, a} Q_{\min }-Q_{\min }}\right)=\frac{1}{1+e^{-c_{F, b}\left(\frac{c_{F, a} Q_{\min }-Q(t)}{c_{F, a} Q_{\min } Q_{\min }}\right)}},
$$

The expression of transporter ${ }_{1}$ is maximum when $Q=Q_{\min }$ and minimum when $Q \gg Q_{\min }$. Note that we replaced the maximum quota $\left(Q_{\max }\right)$ from the Lomas et al. [10] function with $c_{F, a} Q_{\min } \leq Q_{\max }$, which effectively allowed us to calibrate with our experimental data the value of $Q$ at which highaffinity transporters become upregulated. This modification of the $F$ function implied that, as in the case of the growth rate, the gene expression variations of high-affinity transporters mainly occurred at low $Q$ values. Both $c_{F, a}$ and $c_{F, b}$ control the shape of the function. Finally, $H$ is a Heaviside function that limits the synthesis of transporters when the proportion of the cell surface occupied by transporters $\left(A_{r e l}\right)$ surpasses a certain threshold $\left(c_{H}\right): H=0$ if $\sum_{i=1}^{j} A_{\text {rel, },}(t)>c_{H}$, or $H=1$ if $\sum_{i=1}^{j} A_{\text {rel }, i}(t)<c_{H}$, where $\left.\sum_{i=1}^{j} A_{\text {rel }, i}(t)=\sum_{i=1}^{j} n_{i}(t) \frac{r_{s, i}^{2}}{r^{2}}\right)$. Thus, high and low-affinity transporters compete for the cell surface (see SI for further details). 
On the other hand, transporter ${ }_{2}$ is upregulated at high environmental $\mathrm{P}$ levels, enabling the cell to take advantage of $\mathrm{P}$ pulses:

$$
\begin{aligned}
& \frac{d n_{2}(t)}{d t}=v_{2} H\left(c_{H}-\sum_{i=1}^{j} A_{r e l, i}(t)\right) \\
& H\left(\frac{V_{2}(t)}{V_{\max , 2}(t)}-g_{H}\right)-n_{2}(t) \mu(Q, N),
\end{aligned}
$$

where $v_{2}$ is the maximum synthesis rate of transporter 2 . We represent the expression of transporter $_{2}$ as a switch, using a Heaviside function that stops the synthesis of transporters when the ratio $V_{2} / V_{\max , 2}$, the fraction of occupied transporters [31], falls below a threshold $g_{H}$ (see SI for further details). This formulation implies that transporter 2 has both transporter and signaling functions and is therefore considered a transceptor [47]. We assumed that both type of transporters can operate simultaneously. Nonetheless, the model could be modified to take into account the potential occurrence of feedback mechanisms preventing the simultaneous operation of high and low-affinity transporters [47].

Finally, the temporal dynamics of the $\mathrm{P}$ concentration in the medium, affected by the pulse of $\mathrm{P}$ when $\mathrm{t}=6$ days, is provided by:

$\frac{d P}{d t}=-V(t) N(t)$,

The specific values assigned to each parameter and the initial conditions were based on the experimental results and design (see SI text and Table S1). Simulations including only transporter ${ }_{1}$ or transporter ${ }_{2}$ were also run to compare the $V_{\max }$ predictions with those obtained when both transporters were considered.

\section{Results}

\section{Observed dynamics of uptake traits}

Phosphorus-limited cultures of $P$. tricronutum were supplied with different levels of $\mathrm{P}$ pulses $(0,3$, and $15 \mu \mathrm{M} \mathrm{P}$; named $P_{\text {ref }}, P_{\text {low }}$, and $P_{\text {high }}$, respectively). Measuring phosphate uptake traits at three time points following $P$ pulses enabled us to track the dynamics of the acclimation process. The temporal behavior of $V_{\max }$, obtained from P-uptake curves, differed depending on the pulse of P (Fig. 1a). This was confirmed by the significant interaction between $\mathrm{P}$ pulse and Time (ANOVA, $p<0.01$; Table S2). $V_{\max }$ in $P_{r e f}$ was similar along the whole experiment (pairwise contrasts, $p>0.10$; Table S3). On the contrary, $V_{\max }$ showed a V-shape pattern in $P_{\text {low }}$ and a sharp decrease without a subsequent recover in $P_{\text {high }}$ (Fig. 1a). Specifically, $V_{\max }$ was higher in $P_{\text {high }}$ than in $P_{\text {ref }} 3.5 \mathrm{~h}$ after the nutrient pulse (pairwise contrast, $p<$
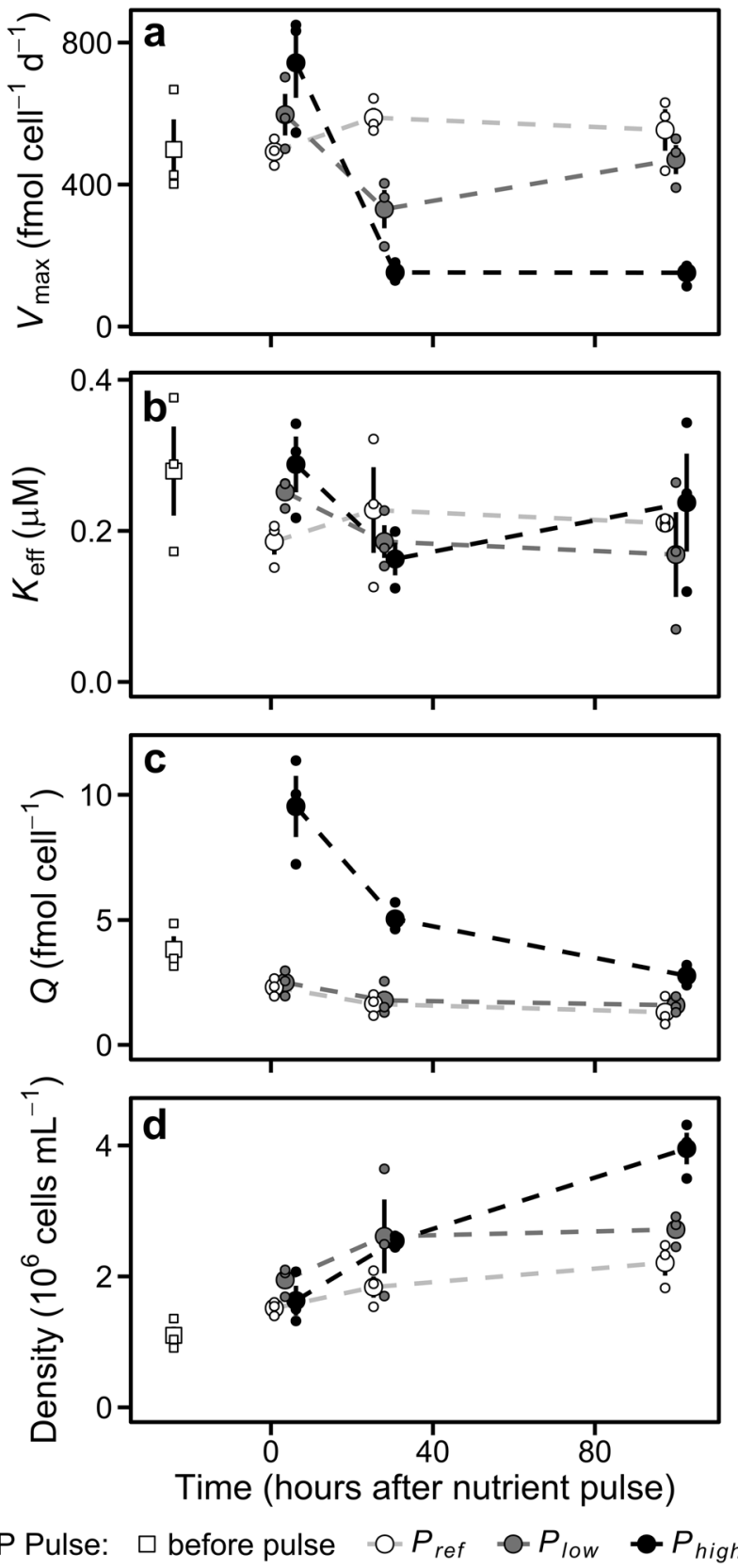

Fig. 1 Dynamics of the population and cellular traits measured in the different $\mathrm{P}$ pulse treatments. a Maximum phosphorus uptake rate $\left(V_{\max }\right)$. b Effective half saturation constant for phosphorus $\left(K_{\text {eff }}\right)$. c Phosphorus quota. d Cell density. Big dots represent mean values. Small dots are the observations from the three experiments. Error bars show standard error estimated from untransformed data. Dashed lines join mean values. The horizontal position of the dots is slightly adjusted to avoid overlapping

0.05). However, $V_{\max }$ notably decreased between 3.5 and 28 $\mathrm{h}$ after the pulse of nutrients in $P_{\text {low }}$ and, more markedly, in $P_{\text {high }}$ (pairwise contrasts, $p<0.01$ ). One hundred hours after the nutrient pulse, $V_{\max }$ in $P_{\text {low }}$ recovered the baseline levels observed in $P_{\text {ref }}$ (pairwise contrast, $p>0.30$ ) but remained low in $P_{\text {high }}$ (pairwise contrast, $p<0.01$ ). 
The dynamics of $K_{\text {eff }}$ are shown in Fig. 1b. We did not observe a significant effect of P pulse on $K_{\text {eff }}$ (ANOVA, $p>$ 0.14 ; Table S2), but we found a marginally significant effect of $\mathrm{P}$ pulse $3.5 \mathrm{~h}$ after the nutrient pulses (ANOVA, $n=9$, $p<0.10)$. As a result of the effect of $\mathrm{P}$ pulse on the dynamics of $V_{\max }$, the $V_{\text {max }}: K_{\text {eff }}$ ratio temporal behavior was also affected (Fig. S5; Table S2).

The addition of $\mathrm{P}$ also affected the dynamics of intracellular phosphorus quota (Fig. 1c). We found a significant interaction between $\mathrm{P}$ pulse and Time (ANOVA, $p<0.05$; Table S2). $3.5 \mathrm{~h}$ after the nutrient pulse $Q$ was higher in $P_{\text {high }}$ than in $P_{\text {ref }}$ (Fig. 1c; pairwise contrast, $p<0.01$; Table S3). Differences in $Q$ among $\mathrm{P}$ pulse treatments decreased with time and were related to changes in $V_{\max }$ (Figs. 1c and S6). Finally, the P pulses also altered population growth, as revealed by the significant effect of $\mathrm{P}$ pulse on population abundances (Fig. 1d; ANOVA, $p<0.01$; Table S2). One hundred hours after the nutrient pulse, cell concentrations were higher in $P_{\text {high }}$ than in $P_{\text {ref }}$ (Fig.1d; pairwise contrast, $p<0.05$; Table S3). This higher growth in $P_{\text {high }}$ contributed to the marked quota decrease observed from 3.5 to $100 \mathrm{~h}$ (Fig. 1c).

\section{Modelled dynamics of uptake traits}

The temporal behavior of $V_{\text {max }}$ predicted by our model with two types of nutrient transporters and different regulation mechanisms matched the patterns observed empirically. The magnitude of the $\mathrm{P}$ pulses affected the in silico $V_{\max }$ dynamics (Fig. 2a). The upregulation of transporter 2 led to a peak in the simulated $V_{\text {max }}$ just after the pulse of $\mathrm{P}$ in both $P_{\text {low }}$ and $P_{\text {high }}$ (Fig. 2c). The $V_{\text {max }}$ peak in $P_{\text {high }}$ was higher than in $P_{\text {low }}$ because the environmental $\mathrm{P}$ concentrations remained for a longer time period above the threshold level $\left(g_{H}\right)$ that keeps low-affinity transporter upregulated (Fig. 2c, f). A high growth rate (Fig. $2 \mathrm{~g}$ ), which reduced the amount of transporters per cell, and the downregulation of high and low-affinity transporters lead to a subsequent $V_{\text {max }}$ decrease (Fig. 2b, c). Both the high growth rate and the downregulation of high-affinity transporters were promoted by the high phosphorus quota (Fig. 2e; Eq. (6)), whereas the downregulation of low-affinity transporters was caused by the low environmental P levels (Fig. 2f; Eq. (7)). This $V_{\max }$ decrease did not occur in $P_{\text {ref }}$, where $Q$ was close to $Q_{\min }$ and $V_{\max , 1}$ rose up to the threshold set by the maximum cell surface area occupied by $\mathrm{P}$ transporters, being the system close to a stationary phase by the end of the simulation (Fig. 2; Eq. (5)).

The decrease in $V_{\max }$ in $P_{\text {low }}$ transitioned to an increase within the first $24 \mathrm{~h}$ after the $\mathrm{P}$ pulse. This $V_{\max }$ recovery was promoted by the upregulation of transporter 1 as a consequence of the reduction of $Q$, and stopped a few hours later when $V_{\max , 1}$ reached the limit set by the maximum cell surface area occupied by uptake sites (Fig. 2a, b, e; Eq. (6)). On the other hand, the long-lasting $V_{\max }$ and $V_{\max , 1}$ decreases in $P_{h i g h}$ ceased at late stages of the simulation as cell depleted $\mathrm{P}$ reserves. Interestingly, the simulations carried out using a version of our model with just transporter ${ }_{1}$ or $_{\text {transporter }}$ were not able to qualitative reproduce all the $V_{\max }$ patterns: a model including only transporter ${ }_{1}$ did not capture the rise in $V_{\text {max }}$ just after $\mathrm{P}$ pulses (Fig. S7a), whereas a model with only transporter $_{2}$ failed to reproduce the recovering in $V_{\max }$ observed in $P_{\text {low }} 100 \mathrm{~h}$ after P pulse (Fig. S7b).

The $K_{\text {eff }}$ peaks observed in $P_{\text {low }}$ and $P_{\text {high }}$ after the pulse of P (Fig. 2d) were fostered by the upregulation of lowaffinity transporters, which show a high $K$, and the increases in diffusion limitation ultimately caused by $V_{\max }$ raises (see Eq. [S5]). The subsequent $K_{\text {eff }}$ decrease and rebound found in both $P_{\text {low }}$ and $P_{\text {high }}$ is explained by variations in diffusion limitation, ultimately resulting from variations in $V_{\max }$ (Fig. 2a, d; see Eq. [S5]). As a result of the effects of the pulses of $\mathrm{P}$ on $V_{\max }$ and $K_{\text {eff }}$ the temporal dynamics of the $V_{\text {max }}: K_{\text {eff }}$ ratio were also affected (Fig. S8).

\section{Gene expression}

In our experiments, all the genes analyzed related to phosphorus metabolism ( $A P$ 49678, CPS 1583), including the three genes coding for $\mathrm{P}$ transporters $(\mathrm{Na} / \mathrm{Pi} 47666, \mathrm{Na} / \mathrm{Pi}$ 47667, and $P i$ 39515), were downregulated in both $P_{\text {low }}$ and $P_{\text {high }}$ just after the $\mathrm{P}$ pulse (Figs. 3 and S9). The stronger gene downregulation in $P_{\text {high }}$ was associated with a higher phosphorus quota, which supports the role assigned to the intracellular $\mathrm{P}$ content in the regulation of transporter ${ }_{1}$ (see $F$ function in methods). Indeed, the decrease in the expression of $\mathrm{Na} / \mathrm{Pi} 47666, \mathrm{Na} / \mathrm{Pi} 47667$, and $\mathrm{Pi} 39515$ transporters within the first $28 \mathrm{~h}$ supports the reduction of $V_{\max }$ empirically observed and $V_{\max , 1}$ predicted by our model (Figs. 1a and 2b). Furthermore, the expression of these transporters in $P_{\text {ref }}$ showed a similar temporal behavior to that reported for $V_{\max }$ and $V_{\max , 1}$. The downregulation of $\mathrm{P}$ transporters in $P_{\text {low }}$ and $P_{\text {high }}$ ceased $100 \mathrm{~h}$ after $\mathrm{P}$ pulses, when the three $\mathrm{P}$ added treatments showed similar expression values (Fig. 3a-c).

\section{Discussion}

We addressed the dynamics of phosphate uptake traits in different $\mathrm{P}$ temporal gradients resulting from pulses of different magnitudes. Our results revealed two opposite $V_{\max ^{-}}$ nutrient trends along the nutrient temporal gradient: in both $P_{\text {high }}$ and $P_{\text {low }}$ we observed an increase in $V_{\max }$ just after the $\mathrm{P}$ pulse, followed by a decrease observed $28 \mathrm{~h}$ after the $\mathrm{P}$ pulse. In the case of $P_{l o w}, V_{\max }$ eventually recovered the values observed before the pulse. These changes in $V_{\max }$ 

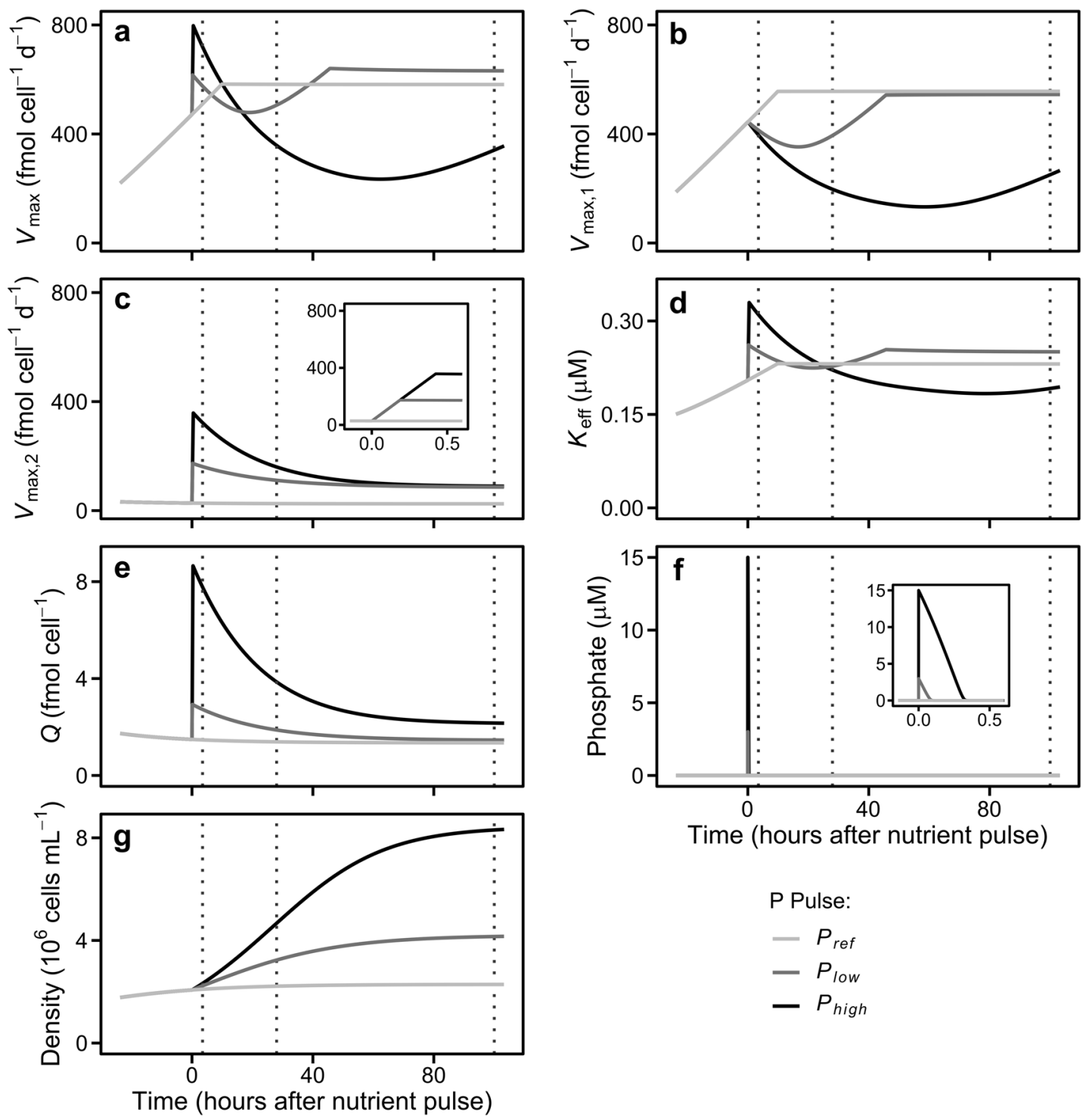

P Pulse:

- Pref

- Plow

- $P_{\text {high }}$

Fig. 2 Dynamics of cellular and population traits predicted by the model in nutrient regimes analogous to those occurring in cultures. a Maximum phosphorus uptake rate $\left(V_{\max }\right)$. b Maximum phosphorus uptake rate for transporter ${ }_{1}$. c Maximum phosphorus uptake rate for $\operatorname{transporter}_{2}\left(V_{\max , 2}\right)$; the inset shows a zoom of the $V_{\max , 2}$ just when the pulses occur (note the different $x$-axis scale). d Effective half

were accompanied by changes in $Q$, and the expression of genes involved in $\mathrm{P}$ uptake and metabolism. Variations in $V_{\max }$ associated with different nutrient conditions have been previously described and modelled, especially those $V_{\max }$ increases occurring under oligotrophic conditions $[6,7,10,29]$. However, to the best of our knowledge, none of these previous studies reported different $V_{\max }$-nutrient patterns along a nutrient continuum and the transition of $V_{\max }$ between opposite trends, nor provided a mechanistic theoretical framework broad enough to integrate the observed diversity of phytoplankton nutrient-uptake acclimation responses. saturation constant for phosphorus $\left(K_{\text {eff }}\right)$. e Phosphorus quota. f Extracellular phosphate concentration; the inset shows a zoom when the pulses occur (note the different $x$-axis scale). g Cell density. To facilitate comparison with empirical results, vertical dotted lines indicate the times when measurements were taken during the experiments

The key feature that allows our mathematical model to reproduce the empirical $V_{\max }$ dynamics is the inclusion of two types of $\mathrm{P}$ transporters with different regulation mechanisms: the synthesis of transporter ${ }_{1}$ (high-affinity) and transporter 2 (low-affinity) depended on intracellular phosphorus quota and $V_{2} / V_{\max , 2}$, respectively. The regulation proposed for transporter 1 was supported empirically by the upregulation at low phosphorus levels of the three genes coding for $\mathrm{P}$ transporters (Fig. 3), and has been widely reported for high-affinity transporters [17, 23, 25, 48]. On the other hand, none of the genes analyzed showed a regulation similar to transporter 2 . The regulation of transporters 


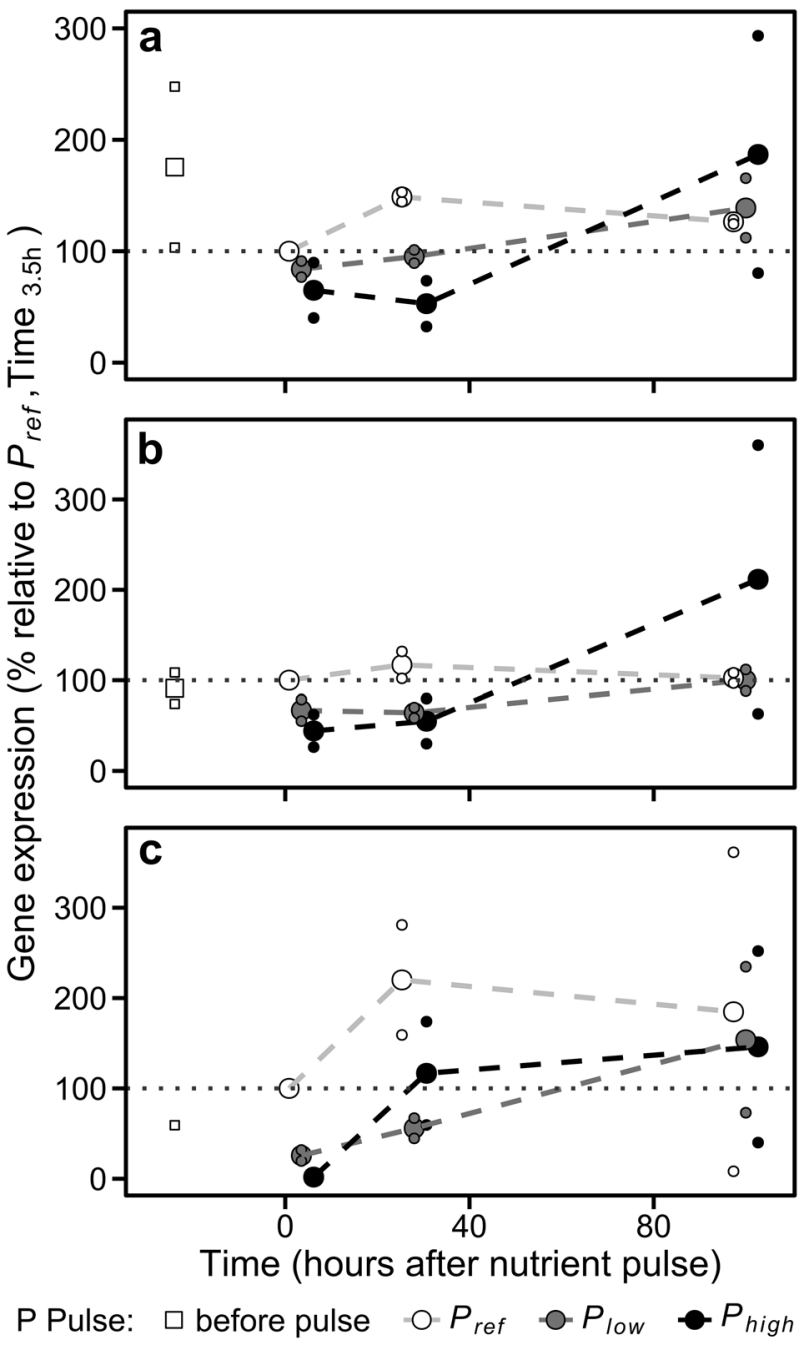

Fig. 3 Gene expression patterns in the different levels of phosphate addition. a $\mathrm{Na} / \mathrm{Pi}$ 47666. b $\mathrm{Na} / \mathrm{Pi}$ 47667. c $\mathrm{Pi}$ 39515. Big dots represent mean values. Small dots are the observations from experiments 2 and 3. Dashed lines join mean values. Grey dotted lines point out the reference level (i.e., 100\%). The horizontal position of the dots is slightly adjusted to avoid overlapping

right after $\mathrm{P}$ pulses in phosphorus-limited phytoplankton cultures has been less analyzed. Nonetheless, Feng et al. [49], in a proteomic analysis in P. tricornutum observed higher levels of a permease involved in $\mathrm{P}$ uptake in phosphorus-replete than in phosphorus-limited conditions. Moreover, a higher activity of low-affinity transporters at high $\mathrm{P}$ concentrations, regulated after transcription, has been reported in yeast $[47,50,51]$. Other possibilities (e.g., high-affinity transporters upregulated at high nutrient levels) previously observed in diatoms for other nutrients like nitrate [52] might occur as well for phoshorus. The existence of transporters with different regulations and kinetics is in agreement with previous works that reported variations in $K_{\text {eff }}$ for species like Cosmarium abbreviatum ([21-23], but see also ref. [19]), and the identification of up to 24 putative $\mathrm{P}$ transporter genes (including mitochondrial carrier proteins) in $P$. tricornutum upregulated at different phosphorus conditions [28]. More than two types of transporters may be present in the cell surface indeed [47], leading to a finer regulation of the $\mathrm{P}$ uptake and a greater capacity to exploit phosphate gradients. Our model provides a simplified version of this transporter diversity, in which transporter ${ }_{1}$ and transporter $_{2}$ can represent clusters of $\mathrm{P}$ uptake proteins upregulated at different phosphorus levels.

The empirical $V_{\max }$ patterns and the model parameterization required to reproduce such patterns suggest differences in the cellular responses to phosphorus limitation and nutrient pulses. For example, the maximum synthesis rate for low-affinity transporters required to reproduce $V_{\text {max }}$ peaks after $\mathrm{P}$ pulses was notably higher than that for high-affinity transporters to reproduce $V_{\max }$ increases at low P levels (unless $K_{2}>3.3 \mu \mathrm{M}$ was used). Analogously, we included different function types (i.e., step vs continuous) to describe the dynamics of both transporters (Eq. (5) and Eq. (7)). The expression of high-affinity transporters occurs at low quotas [23], usually reached after a progressive nutrient depletion; cells with a finer control on the expression of these transporters would make a better use of their internal phosphorus resources and, therefore, would be positively selected. On the contrary, in the case of lowaffinity transporters, which mainly take advantage of high environmental $\mathrm{P}$ concentrations, quick responses might be selected for instead: the cells that respond faster will store more nutrients before they are again depleted, with the efficient use of resources being secondary. Indeed, alternative mechanisms faster than transporter synthesis (e.g., activity regulation of existing uptake sites) have been described in yeast for low-affinity transporters [47, 50]. These ideas about the different velocities and type of responses to phosphorus limitation and pulses somewhat resemble the findings reported by Menge et al. [53], who theoretically predicted that cells showing slow and sensitive plastic responses (i.e., a tortoise-like strategy) prevail in stable environments, whereas cells with fast plastic responses (i.e., a hare-like strategy) prevail in environments showing a higher variability (below certain limits). Interestingly, our results highlight the possibility for the same cell to develop the two strategies, alternating between them thanks to the different regulation of the two types of transporters.

The theoretical model, by considering the temporal dimension and potential mechanisms of the acclimation process, enabled the integration of the snapshots provided by our empirical measurements in a moving picture and broad conceptual framework [30, 31]. In light of our simulations, run under the conditions defined by our experimental design, the $V_{\max }$ and acclimation stage of 


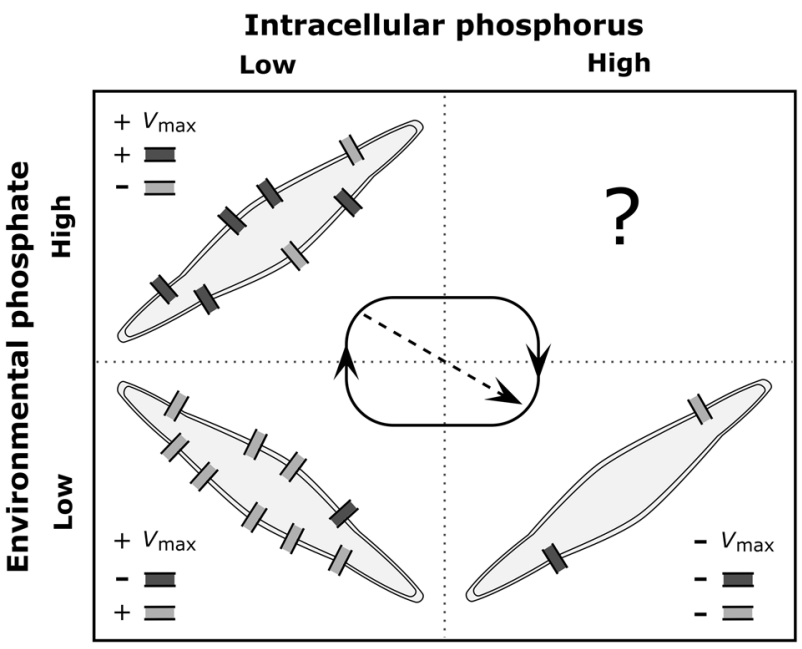

Transporter type: || Low-affinity || High-affinity

Fig. 4 Schematic figure representing the stages of phosphate uptake acclimation in $P$. tricornutum under different nutrient scenarios along the nutrient spatiotemporal gradient. Black arrows indicate the dominant direction of the acclimation succession. The dashed arrow shows the alternative acclimation succession pathway observed in our experiments. The plus sign indicates an increase in trait value or transporter number, whereas minus sign shows a decrease in trait value or transporter number

phosphorus-limited $P$. tricornutum cells can be predicted from environmental and intracellular phosphorus levels, which mainly reflect the present and past nutrient history of the cell, respectively (Fig. S10; refs. [14, 54]). We can indeed classify $P$. tricornutum's uptake acclimation responses along these environmental and intracellular nutrient gradients in four scenarios that can occur sequentially (Fig. 4): (1) low environmental and intracellular phosphorus levels, (2) high environmental $\mathrm{P}$ concentration and low $Q$, (3) low environmental $\mathrm{P}$ concentration and high $Q$, and (4) high environmental and intracellular phosphorus levels (although we did not observe the latter case during our experiments). The scenarios with both environmental and intracellular phosphorus at high or low levels represent homeostasis (i.e., intracellular phosphorus levels matching environmental concentrations) and could lead to stationary dynamics if conditions are prolonged in time. On the contrary, the other two scenarios represent transient situations resulting from environmental nutrient fluctuations and the lag in the cellular physiology. The $V_{\max }$ trends and gene expression patterns observed in our experiments and replicated by our model can therefore be understood as a kind of succession of acclimation responses (Fig. 4; see below for a description of each acclimation stage). Along this succession, the lag in the cell response may lead to a mismatch between gene expression and nutrient-uptake traits, as it occurred during our experiments (e.g., $P_{\text {high }}$ at $100 \mathrm{~h}$ after the P pulse) and it has been suggested for Prochlorococcus sp. [17].
The scenario of high environmental and low intracellular phosphorus levels was observed in $P_{\text {low }}$ and $P_{\text {high }}$ immediately after $P$ pulses. It was characterized by an increase in $V_{\max }$ which, according to our model, occurred due to the upregulation of low-affinity transporters (Figs. 2 and 4). The positive relationship between $V_{\max }$, nutrient transporters, and nutrient levels has been linked to a maximization of the phytoplankton capacity to exploit usually scarce resources [5]. Nevertheless, to the best of our knowledge, our model is the first theoretical effort that mechanistically reproduces the increase in $V_{\max }$ under these conditions. The increase in $Q$ led to the scenario of low environmental and mediumhigh intracellular phosphorus levels, which was observed in $P_{\text {high }}$ after pulse depletion. Here, $V_{\max }$ reached minimum values due to the downregulation of the synthesis of both transporter types (Figs. 1a, 2a and 4). This suggests a cost for the synthesis of transporters and maybe a potential tradeoff with other cell functions: cells invest in nutrient transporters only when it provides an increase in nutrient uptake or cell growth is strongly nutrient limited, otherwise cells invest their resources on growth or the uptake of other nutrients $[53,55]$. Finally, growth caused the draw-down of environmental and intracellular phosphorus levels observed in $P_{\text {ref }}$, and $P_{\text {low }}$ and $P_{\text {high }} 100 \mathrm{~h}$ after P pulses. In this scenario we found an increase in $V_{\max }$ promoted by the upregulation of high-affinity transporters (Figs. 2 and 4). This $V_{\max }$ increase and transport upregulation has been reported in nutrient limited cultures and natural populations of phytoplankton $[6,9,10,17,24,28]$. Such $V_{\max }$ responses can be limited by extreme cellular quota conditions occurring at homeostatic scenarios and systems in a stationary phase. $V_{\max }$ can decrease due to a negative feedback of the internal phosphorus pool on $\mathrm{P}$ uptake or the synthesis of transporters when $Q$ is close to $Q_{\max }$ [12]. Also, Krumhardt et al. [9] reported a marked decrease in $V_{\max }$ in phosphorusstarved ( $Q$ close to $Q_{\min }$ ) cultures of Prochlorococcus sp., not observed for $P$. tricornutum in the present study.

It is plausible that a similar succession of acclimation responses occurs in natural phytoplankton populations experiencing some degree of phosphorus deficiency and environmental variability. Nonetheless, the succession we described could be restricted to those species and ecotypes with both high and low-affinity transporters, as some acclimation strategies might be absent in organisms with just one transporter type. Species and ecotypes with both high and low-affinity transporters would be encountered more often in environments showing high nutrient variations [25], as is the case in the coastal waters inhabited by $P$. triconutum. Indeed, Scanlan et al. [25], in an analysis of several strains of Prochlorococcus and Synechococcus, reported that the only strain with genes coding for potentially both high and low-affinity transporter proteins likely inhabited an estuarine 
environment, whereas the strains from oligotrophic and less variable waters lacked genes coding for low-affinity transporters.

Based on the comparison of the acclimation responses in the three $\mathrm{P}$ pulse treatments, we suggest that the acclimation succession experienced by a specific cell type would depend on the environment. The amplitude of the acclimation succession (i.e., the $V_{\max }$ range in the present context) would tend to decrease from ecosystems (and seasons) with marked temporal nutrient gradients to ecosystems with narrow gradients (Figs. 2 and S10). In addition, the dominant (i.e., most frequent) acclimation stage would change between ecosystems. For example, higher $V_{\max }$ values and the expression of high-affinity transporters as in $P_{\text {ref }}$ would dominate in oligotrophic ecosystems. In this regard, Lomas et al. [10] reported that picocyanobacteria in the Sargasso Sea usually show higher $V_{\max }$ values in summer (strong phosphorus limitation) than in winter. On the contrary, the expression of low-affinity transporters could dominate in eutrophic environments [56]. Other factors like temperature and the availability of non-limiting nutrients might also affect acclimation responses; for instance, temperature may influence the synthesis of transporter proteins [57]. As a result, the succession of acclimation responses occurring at the organismal level might influence the fitness of the cells, the community composition and, therefore, interact with the ecological succession occurring at the community level at wider temporal scales $[4,58]$.

\section{Final remarks}

Although maybe intrinsic to the concept of acclimation, the idea of a temporal sequence of cellular responses might not have been stressed enough in the literature on nutrientuptake acclimation. We believe that understanding acclimation as a succession of responses along the nutrient spatiotemporal gradient offers a novel framework that may contribute to integrating the diverse responses developed by phytoplankton.

Future studies analyzing the dynamics along nutrient spatiotemporal gradients of the traits involved in nutrient uptake in different phytoplankton groups, as well as the expression of genes coding for nutrient transporters, are key to disentangling the diversity of responses and underlying regulation pathways. Sequenced species like Synechococcus sp., Micromonas sp. or Emiliana huxleyi are good candidates. Empirical results could inform mechanistic theoretical models similar to the one proposed here, which could be indeed modified to include alternative or additional regulation mechanisms and transporters, and accommodated to other nutrients or resources. These multidisciplinary studies would chart a way forward to assess the ecological importance of acclimation and bridge the gap between cellular physiology, community ecology, and evolution.

Acknowledgements We thank the members of the Marine Resource Modeling group at Strathclyde University, specially M.R. Heath and M. Choua, for their comments on the model and our preliminary results. We would also like to acknowledge K. Griffiths for her help with ${ }^{33} \mathrm{P}$ handling. M. W. Lomas kindly advised on the methodology for measuring P uptake. Suggestions and comments provided by James Grover and discussions with R. Anadón, F.G. Taboada, S. RomeroRomero and R. González-Gil are greatly appreciated. This research has been supported by the Marine Alliance for Science and Technology for Scotland (MASTS). C.C., S.S., and J.A.B. were supported by the Marine Alliance for Science and Technology for Scotland (MASTS) pooling initiative, funded by the Scottish Funding Council (HR09011) and contributing institutions. E.K. is grateful to the John Grieve Bequest and the Biotechnology and Biological Sciences Research Council (BBSRC) for the New Investigator Grant Award BB/ M023079/1.

Funding This research has been supported by the Marine Alliance for Science and Technology for Scotland (MASTS). C.C., S.S., and J.A.B. were supported by the Marine Alliance for Science and Technology for Scotland (MASTS) pooling initiative, funded by the Scottish Funding Council (HR09011) and contributing institutions. Gene expression experiments and analysis performed by E.K. were funded by the Biotechnology and Biological Sciences Research Council (BBSRC) (Grant Award BB/M023079/1).

\section{Compliance with ethical standards}

Conflict of interest The authors declare that they have no conflict of interest.

Publisher's note: Springer Nature remains neutral with regard to jurisdictional claims in published maps and institutional affiliations.

\section{References}

1. Falkowski PG, Barber RT, Smetacek V. Biogeochemical controls and feedbacks on ocean primary production. Science. 1998;281:200-6.

2. Field CB, Behrenfeld MJ, Randerson JT, Falkowski P. Primary production of the biosphere: integrating terrestrial and oceanic components. Science. 1998;281:237-40.

3. Reynolds CS. The ecology of phytoplankton. Cambridge University Press; Cambridge, UK: 2006.

4. Reynolds C, Dokulil M, Padisák J. Understanding the assembly of phytoplankton in relation to the trophic spectrum: where are we now? Hydrobiologia. 2000;424:147-52.

5. Caperon J, Meyer J. Nitrogen-limited growth of marine phytoplankton-II. Uptake kinetics and their role in nutrient limited growth of phytoplankton. Deep-Sea Res. 1972;19:619-32.

6. Gotham IJ, Rhee G-Y. Comparative kinetic studies of phosphatelimited growth and phosphate uptake in phytoplankton in continuous culture. J Phycol. 1981;17:257-65.

7. Morel FMM. Kinetics of nutrient uptake and growth in phytoplankton. J Phycol. 1987;23:137-50.

8. Zhang S-F, Yuan C-J, Chen Y, Chen X-H, Li D-X, Liu J-L, et al. Comparative transcriptomic analysis reveals novel insights into the adaptive response of Skeletonema costatum to changing ambient phosphorus. Front Microbiol. 2016;7:1476. 
9. Krumhardt KM, Callnan K, Roache-Johnson K, Swett T, Robinson D, Reistetter EN, et al. Effects of phosphorus starvation versus limitation on the marine cyanobacterium Prochlorococcus MED4 I: uptake physiology. Environ Microbiol. 2013;15:2114-28.

10. Lomas MW, Bonachela JA, Levin SA, Martiny AC. Impact of ocean phytoplankton diversity on phosphate uptake. Proc Natl Acad Sci USA. 2014;111:17540-5.

11. Sunda WG, Huntsman SA. Regulation of cellular manganese and manganese transport rates in the unicellular alga Chlamydomonas. Limnol Oceano. 1985;30:71-80.

12. Riegman R, Stolte W, Noordeloos AAM, Slezak D. Nutrient uptake and alkaline phosphatase (EC 3: 1: 3: 1) activity of Emiliania huxleyi (Prymnesiophyceae) during growth under $\mathrm{N}$ and $\mathrm{P}$ limitation in continuous cultures. J Phycol. 2000;36:87-96.

13. Michaelis L, Menten ML. Die kinetik der invertinwirkung. Biochem Z. 1913;49:333-69.

14. Droop MR. Vitamin $B_{12}$ and marine ecology. IV. The kinetics of uptake, growth and inhibition in Monochrysis lutheri. J Mar Biol Assoc U Kingd. 1968;48:689-733.

15. Aksnes DL, Egge JK. A theoretical model for nutrient uptake in phytoplankton. Mar Ecol Prog Ser. 1991;70:65-72.

16. Litchman E, Klausmeier CA. Trait-based community ecology of phytoplankton. Annu Rev Ecol Evol Syst. 2008;39:615-39.

17. Reistetter EN, Krumhardt K, Callnan K, Roache-Johnson K, Saunders JK, Moore LR, et al. Effects of phosphorus starvation versus limitation on the marine cyanobacterium Prochlorococcus MED4 II: gene expression. Environ Microbiol. 2013;15:2129-43.

18. Healey FP. Slope of the Monod equation as an indicator of advantage in nutrient competition. Micro Ecol. 1980;5:281-6.

19. Fu F, Zhang Y, Bell PRF, Hutchins DA. Phosphate uptake and growth kinetics of Trichodesmium (Cyanobacteria) isolates from the North Atlantic Ocean and the Great Barrier Reef, Australia. J Phycol. 2005;41:62-73.

20. Collos Y, Vaquer A, Souchu P. Acclimation of nitrate uptake by phytoplankton to high substrate levels. J Phycol 2005;41:466-78.

21. Rivkin RB, Swift E. Phosphate uptake by the oceanic dinoflagellate Pyrocystis noctiluca. J Phycol. 1982;18:113-20.

22. Spijkerman E, Coesel PFM. Phosphorus uptake and growth kinetics of two planktonic desmid species. Eur J Phycol. 1996;31:53-60.

23. Lin S, Litaker RW, Sunda WG. Phosphorus physiological ecology and molecular mechanisms in marine phytoplankton. J Phycol. 2016;52:10-36.

24. Martiny AC, Coleman ML, Chisholm SW. Phosphate acquisition genes in Prochlorococcus ecotypes: evidence for genome-wide adaptation. Proc Natl Acad Sci USA. 2006;103:12552-7.

25. Scanlan DJ, Ostrowski M, Mazard S, Dufresne A, Garczarek L, Hess WR, et al. Ecological genomics of marine picocyanobacteria. Microbiol Mol Biol Rev. 2009;73:249-99.

26. Dyhrman ST, Jenkins BD, Rynearson TA, Saito MA, Mercier $\mathrm{ML}$, Alexander $\mathrm{H}$, et al. The transcriptome and proteome of the diatom Thalassiosira pseudonana reveal a diverse phosphorus stress response. PLoS ONE. 2012;7:e33768.

27. Liu Z, Koid AE, Terrado R, Campbell V, Caron DA, Heidelberg KB. Changes in gene expression of Prymnesium parvum induced by nitrogen and phosphorus limitation. Front Microbiol. 2015;6:631.

28. Cruz de Carvalho MH, Sun H, Bowler C, Chua N. Noncoding and coding transcriptome responses of a marine diatom to phosphate fluctuations. New Phytol. 2016;210:497-510.

29. Smith SL, Yamanaka Y, Pahlow M, Oschlies A. Optimal uptake kinetics: physiological acclimation explains the pattern of nitrate uptake by phytoplankton in the ocean. Mar Ecol Prog Ser. 2009;384:1-12.

30. Dick GJ. Embracing the mantra of modellers and synthesizing omics, experiments and models. Environ Microbiol Rep. 2017;9: $18-20$.
31. Bonachela JA, Raghib M, Levin SA. Dynamic model of flexible phytoplankton nutrient uptake. Proc Natl Acad Sci USA. 2011;108:20633-8.

32. Button DK. Nutrient uptake by microorganisms according to kinetic parameters from theory as related to cytoarchitecture. Microbiol Mol Biol Rev. 1998;62:636-45.

33. Mann KH, Lazier JRN. Dynamics of marine ecosystems: biological-physical interactions in the oceans. John Wiley \& Sons; Oxford, UK; 2013.

34. Visser M, Batten S, Becker G, Bot P, Colijn F, Damm P, et al. Time series analysis of monthly mean data of temperature, salinity, nutrients, suspended matter, phyto-and zooplankton at eight locations on the Northwest European shelf. Dtsch Hydrogr Z. 1996;48:299-323.

35. Blauw AN, Beninca E, Laane RWPM, Greenwood N, Huisman J. Dancing with the tides: fluctuations of coastal phytoplankton orchestrated by different oscillatory modes of the tidal cycle. PLoS One. 2012;7:e49319.

36. Tovar-Sanchez A, Sañudo-Wilhelmy SA, Garcia-Vargas M, Weaver RS, Popels LC, Hutchins DA. A trace metal clean reagent to remove surface-bound iron from marine phytoplankton. Mar Chem. 2003;82:91-9.

37. Siaut M, Heijde M, Mangogna M, Montsant A, Coesel S, Allen A, et al. Molecular toolbox for studying diatom biology in Phaeodactylum tricornutum. Gene. 2007;406:23-35.

38. Vandesompele J, De Preter K, Pattyn F, Poppe B, Van Roy N, De Paepe A, et al. Accurate normalization of real-time quantitative RT-PCR data by geometric averaging of multiple internal control genes. Genome Biol. 2002;3:0034.1-0034.12.

39. Kaiserli E, Paldi K, O'Donnell L, Batalov O, Pedmale UV, Nusinow DA, et al. Integration of light and photoperiodic signaling in transcriptional nuclear foci. Dev Cell. 2015;35: 311-21.

40. Quinn GP, Keough MJ. Experimental design and data analysis for biologists. Cambridge University Press; Cambridge, UK; 2002.

41. R Core Team. R: a language and environment for statistical computing. R Foundation for Statistical Computing, Vienna, Austria. 2017. http://www.R-project.org/

42. Pinheiro J, Bates D, DebRoy S, Sarkar D, Heisterkamp S, Van Willigen B, et al. Package "nlme." Linear nonlinear Mix Eff Model. 2017; R package version 3.1-131. https://CRAN.Rproject.org/package $=$ nlme

43. Lenth R. Emmeans: estimated marginal means, aka least-squares means. 2018; R Package Version 1.3.0. https://CRAN.R-project. org/package $=$ emmeans

44. Benjamini Y, Hochberg Y. Controlling the false discovery rate: a practical and powerful approach to multiple testing. J R Stat Soc Ser B. 1995;57:289-300.

45. Molina Grima E, García Camacho F, Sanchéz Pérez JA, Fernández Sevilla JM, Acién Fernández FG, Contreras Gómez A. A mathematical model of microalgal growth in light-limited chemostat culture. J Chem Technol Biotechnol Int Res Process Environ Clean Technol. 1994;61:167-73.

46. Olli K, Trunov K. Self-toxicity of Prymnesium parvum (Prymnesiophyceae). Phycologia. 2007;46:109-12.

47. Secco D, Wang C, Shou H, Whelan J. Phosphate homeostasis in the yeast Saccharomyces cerevisiae, the key role of the SPX domain-containing proteins. FEBS Lett. 2012;586:289-95.

48. Dyhrman ST. Nutrients and their acquisition: phosphorus physiology in microalgae. In: The physiology of microalgae. MA Borowitzka, J Beardall, JA Raven, editors. Dordrecht, The Netherlands. Springer; 2016. p. 155-83.

49. Feng T-Y, Yang Z-K, Zheng J-W, Xie Y, Li D-W, Murugan SB, et al. Examination of metabolic responses to phosphorus limitation via proteomic analyses in the marine diatom Phaeodactylum tricornutum. Sci Rep. 2015;5:10373. 
50. Hürlimann HC, Pinson B, Stadler-Waibel M, Zeeman SC, Freimoser FM. The SPX domain of the yeast low-affinity phosphate transporter Pho90 regulates transport activity. EMBO Rep. 2009;10:1003-8.

51. Ghillebert R, Swinnen E, De Snijder P, Smets B, Winderickx J. Differential roles for the low-affinity phosphate transporters Pho87 and Pho90 in Saccharomyces cerevisiae. Biochem J. 2011;434:243-51.

52. Song B, Ward BB. Molecular cloning and characterization of high-affinity nitrate transporter in marine phytoplankton. J Phycol. 2007;43:542-52.

53. Menge DNL, Ballantyne F, Weitz JS. Dynamics of nutrient uptake strategies: lessons from the tortoise and the hare. Theor Ecol. 2011;4:163-77.

54. Perry MJ. Phosphate utilization by an oceanic diatom in phosphorus-limited chemostat culture and in the oligotrophic waters of the central North Pacific. Limnol Oceano. 1976;21: 88-107.

55. Klausmeier CA, Litchman E, Levin SA. A model of flexible uptake of two essential resources. J Theor Biol. 2007; 246:278-89.

56. Zielinski BL, Allen AE, Carpenter EJ, Coles VJ, Crump BC, Doherty M, et al. Patterns of transcript abundance of eukaryotic biogeochemically-relevant genes in the Amazon River Plume. PLoS ONE. 2016;7:e0160929.

57. Toseland A, Daines SJ, Clark JR, Kirkham A, Strauss J, Uhlig $\mathrm{C}$, et al. The impact of temperature on marine phytoplankton resource allocation and metabolism. Nat Clim Chang. 2013;3: 979-84.

58. Margalef R. Life-forms of phytoplankton as survival alternatives in an unstable environment. Oceano acta. 1978;1:493-509. 